\title{
Molecular Docking and Analysis of Survivin Delta-Ex3 Isoform Protein
}

\author{
Z. Ezziane*
}

Department of Information Technology, Higher Colleges of Technology, Al Ain Women's College, Al Ain, P.O. Box 17258, United Arab Emirates

\begin{abstract}
This project explores molecular models of Survivin Delta-Ex3, H-Ras, and their binding sites, and generates energy optimized 3D coordinates of docked poses and conformations of the XY2 ligand molecule in the active site of Delta-Ex3. The aim is to propose an effective anti-cancer drug that induces apoptosis and inhibits tumor angiogenesis.
\end{abstract}

\section{INTRODUCTION}

Wikberg and co-workers [1] are applying proteochemometrics as a bioinformatics entity to conduct biomedical research and drug design. This newly developed technology for functional genomics analysis has the potential to be applied over entire genomes and proteomes. Molecular modeling and chemoinformatics represent a crucial factor of drug discovery in every pharmaceutical industry. Rational drug design methods accelerate the process by speeding up the discovery of new chemical entities that may become new drugs. Structure-based design shows precisely the location and orientation of bound inhibitors and describes the physicochemical properties.

Survivin is a baculovirus inhibitor of apoptosis (IAP) repeat (BIR) motif protein and has essential roles in mitosis and cell cleavage. When overexpressed as it is in cancer cells, survivin is present in interphase during which is reported to suppress caspase-3 activity. It is expressed generally during normal embryonic development, but only in a small subset of adult normal differentiated tissues, including the colonic epithelium, uterine endometrium, vascular endothelium, and the subventricular region of normal brain [2-5].

It was reported that survivin's function in cancer is mainly as an IAP, blocking mitochondrial-dependent apoptosis $[6,7]$. However, survivin is also reported later to have a role in a mitotic checkpoint as a chromosomal passenger protein [8]. This family of proteins aligns the chromosomes properly during mitosis and maintains accurate cell division in normal cells [9]. It also avoids the development of abnormal numbers of chromosomes that may occur during the transition from a nonmalignant to a malignant phenotype [10].

There are several survivin isoforms including 2B, 3B, 2alpha, and Delta-Ex3 [11]. These proteins are directly involved in apoptosis. Delta-Ex3 is also found to have a role in angiogenesis [12].

Ras is a small GTPase (regulatory hydrolase) wherein healthy cells shuttles between on and off states - Activated (RAS-GTP) by guanine exchange factors (GEFs) and inactivated (RAS-GDP) by RasGAP [13]. Ras proteins are essen-

*Address correspondence to this author at the Higher Colleges of Technology, Al Ain Women's College, Department of IT, P.O. Box 17258, Al Ain, United Arab Emirates; Tel: + 97137095 229; Fax: + 9713782 0766;

E-mail: zoheir.ezziane@hct.ac.ae tial for proliferation, cell adhesion, apoptosis, and cell migration. When Ras is not properly regulated, it also plays a role in proliferation and malignant transformation (decreased apoptosis). H-Ras is a GTPase in the Ras family which has a role in various signal transduction pathways and is generally linked with cell membranes due to the presence of an isoprenyl group on its c-terminus [14-16]. Once H-Ras is activated (turned on) it recruits and activates proteins needed for the propagation of the receptor's signal such as c-Raf. H-Ras binds to GTP in the active state and holds a fundamental enzymatic activity that cleaves the terminal phosphate of this nucleotide converting it to GDP, which ultimately turn off H-Ras.

Causative relations and interactions existing among survivin proteins and $\mathrm{H}$-Ras will provide additional data to comprehend the possibility of designing effective anti-cancer drugs.

\section{BACKGROUND: RAS PATHWAY AND APOPTOTIC PROTEINS}

Ras proteins are also essential for numerous signal pathways that control processes such as proliferation and apoptosis. Ras activates serine/threonine protein kinase Raf, which in turn activates another serine/threonine protein kinase MEK that triggers MAPK. Raf, MEK, and MAPK are considered mitogen-activated kinases. The deregulation of Ras which leads to increased metastasis (movement of cancerous cells) and decreased apoptosis often results in cancers. Since Ras leads to numerous tumors, then it would be valuable to discover a drug that is able to impose regulation into the Ras pathway, or eradicate cells with uncontrolled Ras pathways. Regulation of pro-apoptotic proteins under normal cell conditions of non-apoptotic cells is not understood entirely. As a result molecules that regulate apoptosis are being studied as potential targets for anticancer drug therapies. A MAPK pathway is shown in Fig. (1).

Since H-Ras is known to down-regulate survivin [17] and Delta-Ex3 has a role in angiogenesis [12], this work is then set to explore potential interactions between Delta-Ex3 and $\mathrm{H}-\mathrm{Ras}$ in order to design apoptotic-based and angiogenesisbased anti-cancer drugs. 


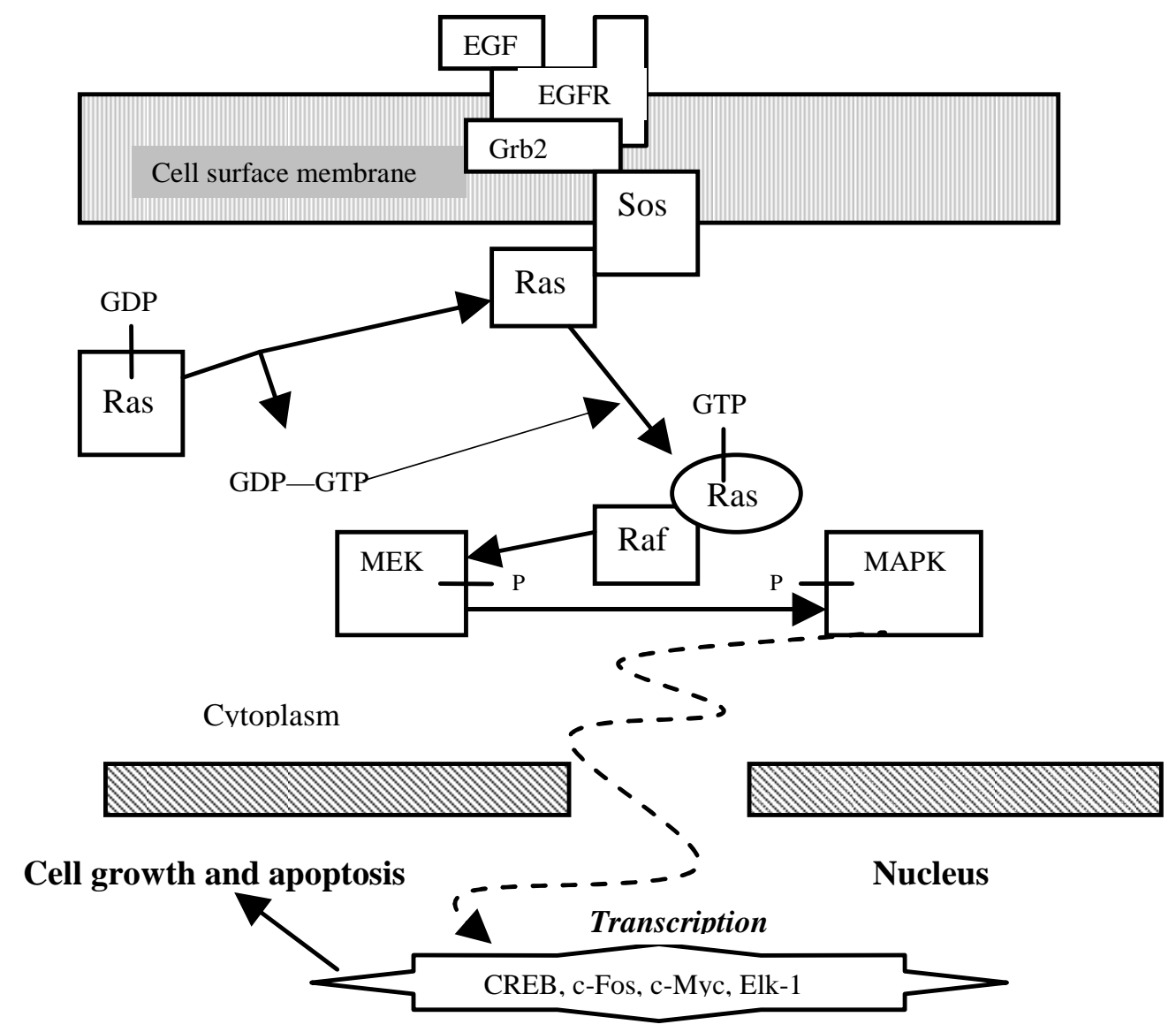

Fig. (1). MAPK Pathway.

\section{COMPUTATIONAL METHODS: ROADMAP OF E- DRUG DESIGN AND DISCOVERY}

Several software tools and "omic" databases are used to design a potential lead composed of a receptor and a ligand. Tools used in this work include Chimera [18], SimDOCK [19], PocketDepth [20], and e-HiTS [21]. Protein databases used in this study include PDB [22] and Swiss-Prot [23].

$\mathrm{H}-\mathrm{R}$ as and its ligands $\mathrm{CAG}$ and $\mathrm{XY} 2$ are shown in Fig. (2). CAG and XY2 appear clearly on top center and top left of Fig. (2). Delta-Ex3 is depicted in Fig. (3).
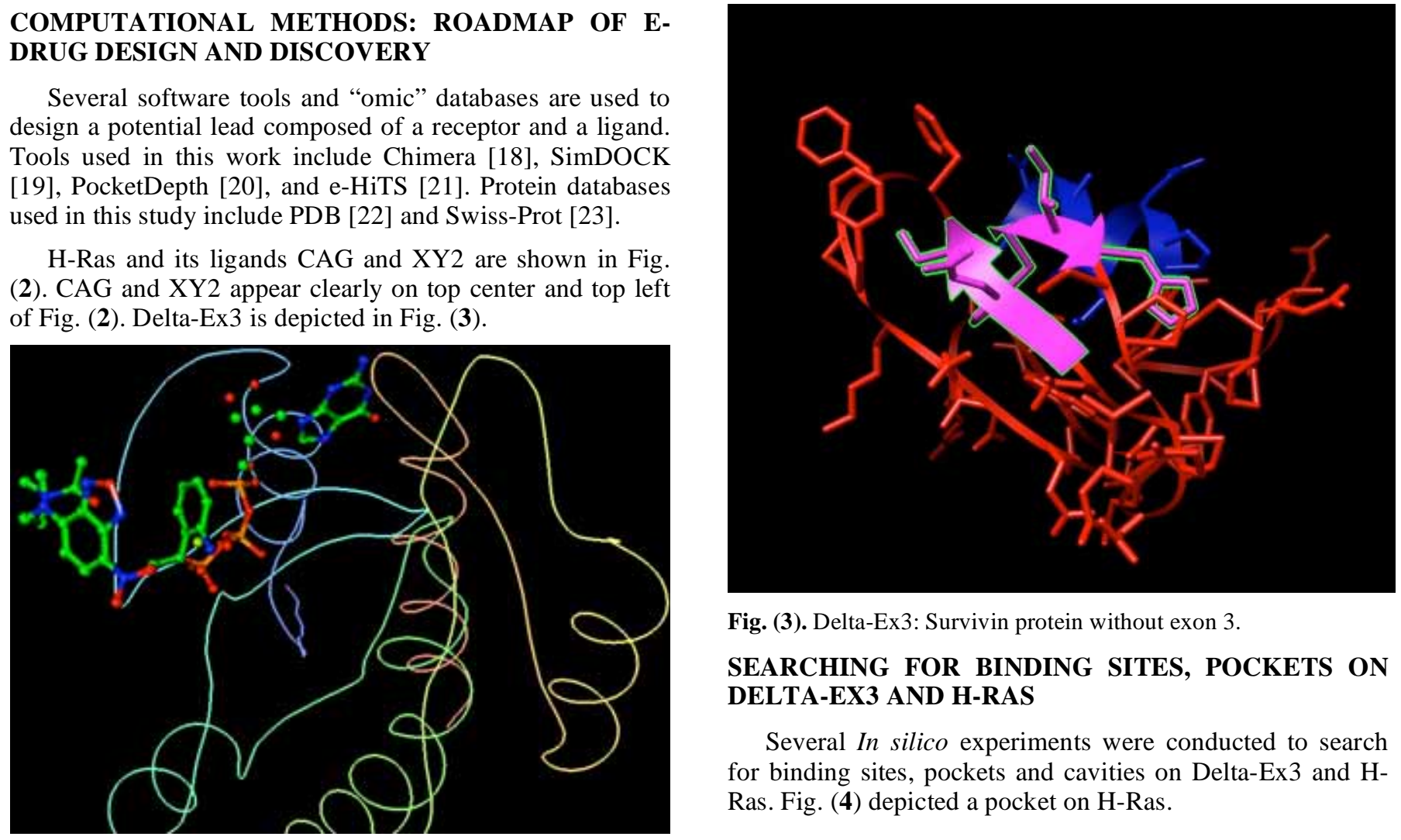

Fig. (3). Delta-Ex3: Survivin protein without exon 3.

\section{SEARCHING FOR BINDING SITES, POCKETS ON DELTA-EX3 AND H-RAS}

Several In silico experiments were conducted to search for binding sites, pockets and cavities on Delta-Ex3 and HRas. Fig. (4) depicted a pocket on H-Ras.

Fig. (2). H-Ras and its ligands CAG and XY2. 


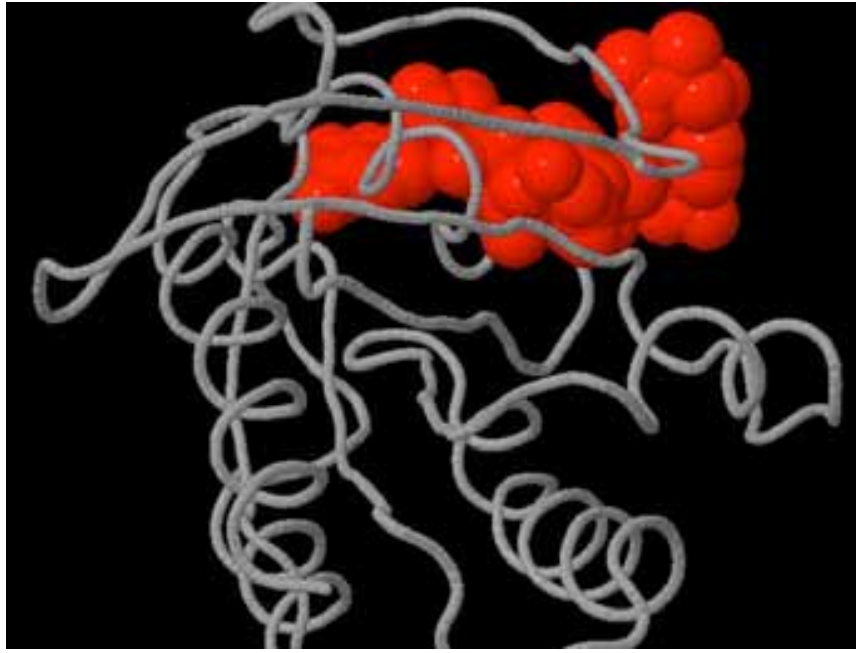

Fig. (4). Pocket (shown in red) on H-Ras.

Various pockets were found on Delta-Ex3. Fig. (5) shows one pocket clearly visible in white.

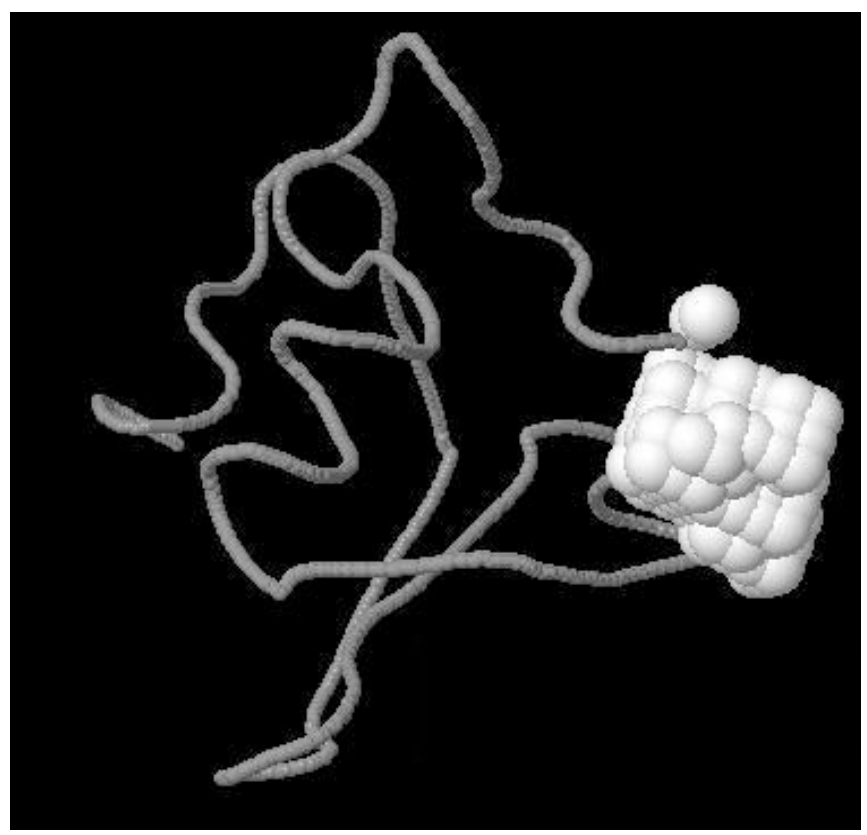

Fig. (5). Pocket (shown in white) on Delta-Ex3.

The next experiments focused on studying the possibility of interacting Delta-Ex3 with ligands of H-Ras, i.e. CAG and $\mathrm{XY} 2$ shown in Figs. $(\mathbf{6 , 7 )}$ respectively. The resulting complexes generated from the In silico experiments represent the expected hits for future anti-cancer drug investigation.

\section{DOCKING AND ANALYSIS}

Molecular docking is used here to predict the conformation, orientation, and poses of the ligand XY2 within the active site of survivin Delta-Ex3 in order to form a stable complex. Knowing the preferred orientation could be used to predict the strength of association and/or binding affinity between XY2 and Delta-Ex3 using scoring functions. Chimera and e-HiTS tools are used to perform many of the following steps. The computational procedures were carried out on an HP computer with an Intel Pentium 4 processor, 2 $\mathrm{GHz}$ and 2 GB RAM.

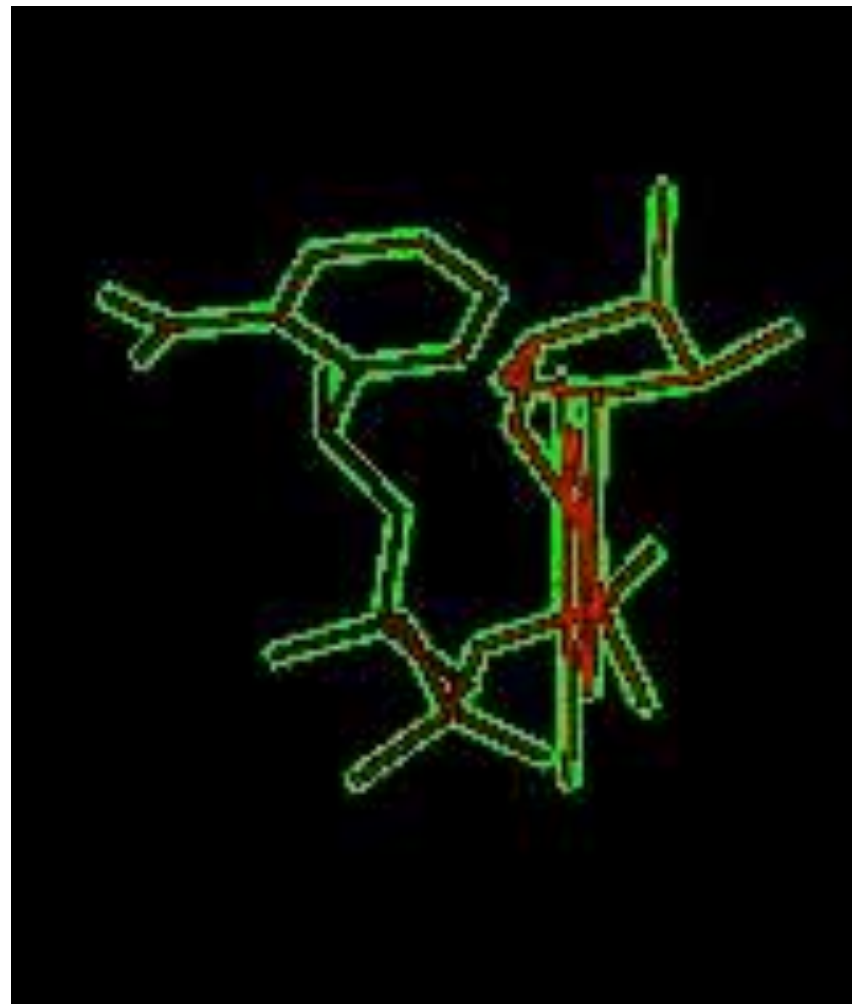

Fig. (6). Ligand CAG of H-Ras.

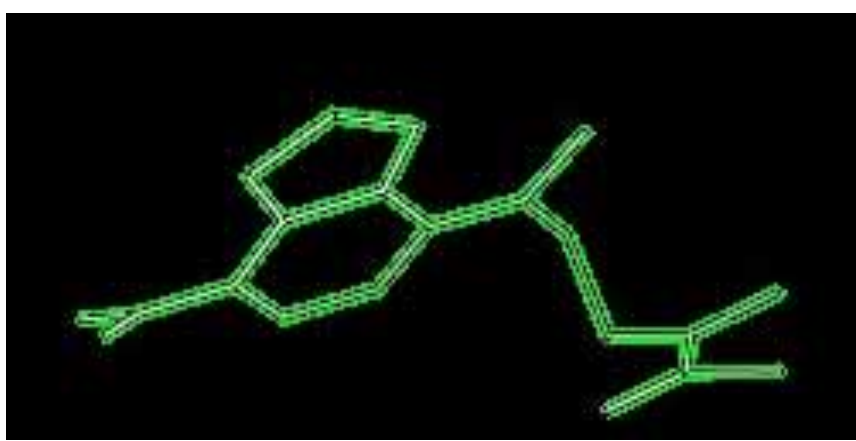

Fig. (7). Ligand XY2 of H-Ras.

1. Protein ("Receptor") input file preparation: Using the survivin Delta-Ex3 protein structure (PDB code: 1E31B), a "clean input file" was generated by removing water molecules, ions, and subunits not involved in ligand binding from the original structure file. Subsequently, hydrogen atoms were added to the receptor and the active site was inspected to make suitable corrections of protonation states of charged residues. Local minimization was then performed in the presence to relieve potential bad contacts, at the same time maintaining the protein conformation as close as possible to that observed in the crystallographic model. The resulting receptor model was saved to a PDB file.

2. Ligand input file preparation: The ligand XY2 was removed from $\mathrm{H}-\mathrm{Ras}$ protein (PDB code: $2 \mathrm{EVW}$ ) and its structure was saved to a PDB file.

3. e-HiTS docking tool: 32 potential binding results were found when ligand XY2 was docked into DeltaEx3. It took 4 minutes to complete the entire process 


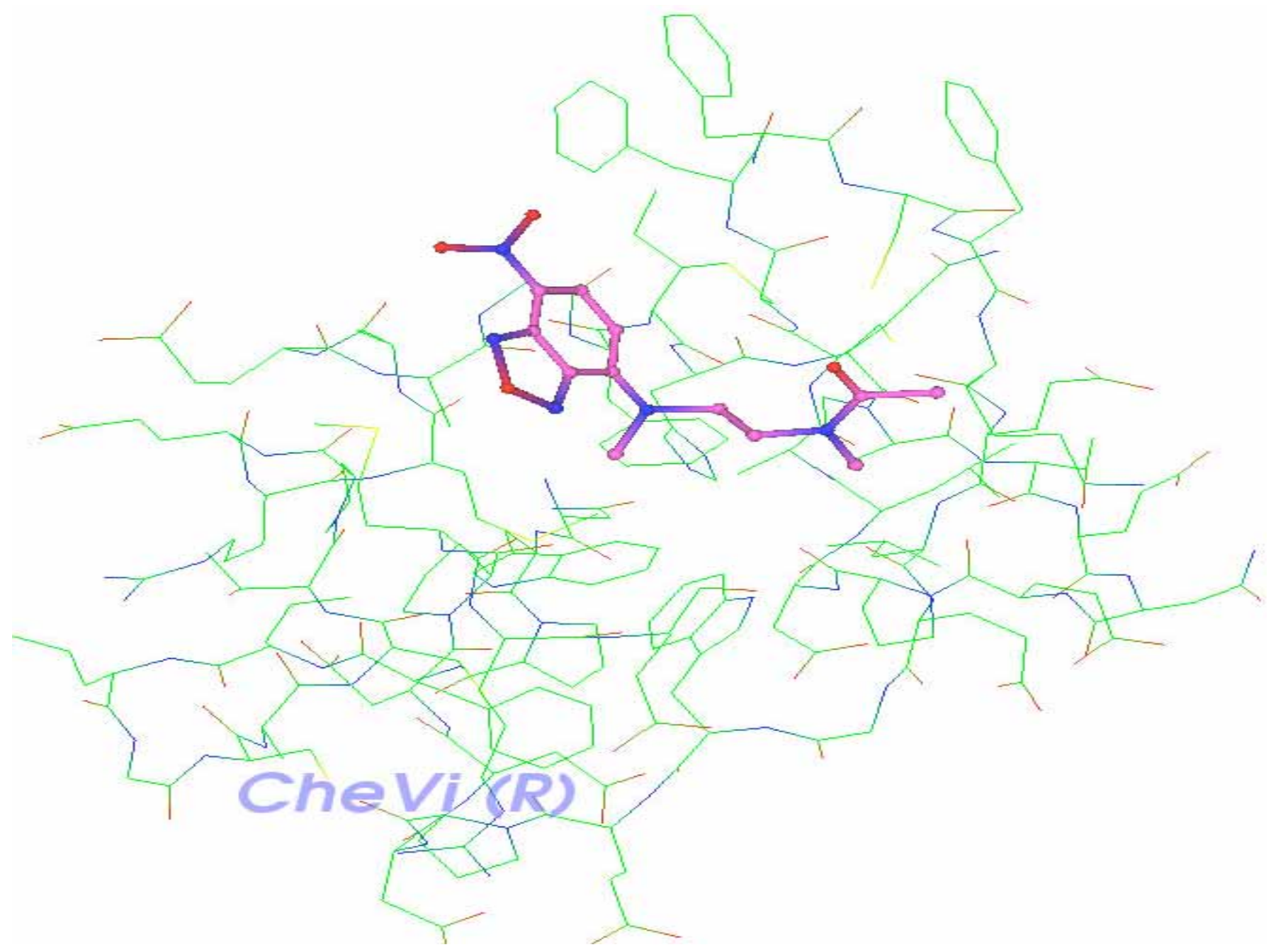

Fig. (8). Complex receptor-ligand: Delta-Ex3 and XY2.

and the best score was -3.247 which includes terms describing the inter- and intramolecular energies.

XY2 ligand is shown docked along Delta-Ex3's surface and deeply embedded in a particular active site (Fig. 8).

\section{CONCLUDING REMARKS AND FUTURE WORK}

Caldas and his team [11] reported that Delta-Ex3 is found to have a role in angiogenesis, and consequently, this paper attempted to identify a possible cell signaling that induces apoptosis and inhibits angiogenesis through survivin isoform Delta-Ex3. In addition, H-Ras was found to down-regulate Delta-Ex3 [15], thus, potential interactions between a ligand of H-Ras and Delta-Ex3 are explored in this paper. Fig. 7 illustrates such possible interactions between Delta-Ex3 and HRas, where a binding of the HRas ligand XY2 is deeply embedded on Delta-Ex3's surface. Additional lab experiments would elucidate the corresponding signaling process vis-à-vis apoptosis and angiogenesis.

The identified complex "Delta-Ex3 and XY2" shown in figure 7 serves as a protein complex that requires further In Silico processing including lead structure optimization. This information could assists researchers in designing drug compounds that bind selectively and tightly onto survivin DeltaEx3..
The next phase of this project is to improve the design of survivin-based anti-cancer drug that induces apoptosis and inhibits tumor angiogenesis through the design of a multipeptide link between XY2 and Delta-Ex3 [24-26].

\section{REFERENCES}

[1] Proteochemometrics.org - A Site Dedicated to the Research of Proteochemometrics. http://www.proteochemometrics.org/ (accessed March 12, 2008).

[2] Konno, R.; Yamakawa H.; Utsunomiya, H.; Ito, K.; Sato, S.; Yajima, A. Mol. Hum. Reprod., 2000, 6, 529.

[3] Gianani, R.; Jarboe, E.; Orlicky, D.; Frost, M.; Bobak, J.; Lehner, R.; Shroyer, K.R. Hum. Pathol., 2001, 32, 119.

[4] Altieri, D. Nat. Rev. Cancer, 2003, 3, 46.

[5] Altura, R.A.; Olshefski, R.; Jiang, Y.; Boue, D.R. Br. J. Cancer, 2003, 89, 1743.

[6] Li, F.; Ambrosini, G.; Chu, E.Y.; Plescia, J.; Tognin, S.; Marchisio, P.C.; Altieri, D.C. Nature, 1998, 396, 580.

[7] Altieri, D.C.; Marchisio, C. Lab Invest., 1999, 79,1327.

[8] Colnaghi, R.; Connell, C.M.; Barrett, R.M.A.; Wheatley, S.P. J. Bio. Chem., 2006, 44, 33450.

[9] Lens, S.M.; Wolthuis, R.M.; Klompmaker, R.; Kauw, J.; Agami, R.; Brummelkamp, T.; Kops, G.; Medema, R.H. EMBO J., 2003, 22, 2934.

[10] Fangusaro, J.R.; Jiang Y.; Holloway, M.P.; Caldas, H.; Singh V.; Boue', D.R.; Hayes, J.; Altura, R.A. Br. J. Cancer, 2005, 92, 359.

[11] Caldas, H.; Honsey, L.E.; Altura, R.A. Mol. Cancer, 2005, 4, 1.

[12] Caldas, H.; Fangusaro, J.R.; Boue, D.R.; Holloway, M.P.; Altura, R.A. Boold, 2007, 104, 1479. 
[13] Reuter C.; Morgan M.; Bergmann L. Blood, 2000, 96, 5, 1655.

[14] Mascaux, C.; Iannino, N.; Martin, B. Paesmans, M.; Berghmans, T. ; Dusart, M. ; Haller, A. ; Lothaire, P. ; Meert, A.P. ; Noel, S. ; Lafitte, J.J. ; Sculier, J.P. Br. J. Cancer, 2005, 92, 131.

[15] Tennant, R.W.; Stasiewicz, S.; Eastin, W.C. ; Mennear, J.H.; Spalding, J.W. Toxicol. Pathol., 2001, 29, 1.

[16] Ayllón, V.; Rebollo A. Mol. Membr. Biol., 2001, 17, 2, 65.

[17] Sommer, K.W.; Rodgarkia-Dara, C.J.; Schreiner, C.; Holzmann, K.; Krupitza, G.; Cerni, C. FEBS Lett., 2007, 17904124.

[18] UCSF Chimera - An Extensible Molecular Modeling System. http://www.cgl.ucsf.edu/chimera/ (accessed October 11, 2007).

[19] SimDOCK. http://sourceforge.net/projects/simdock/ (accessed January 11,2008$)$.
[20] PocketDepth - A New Depth Based Algorithm for Identification of Ligand Binding Sites. http://proline.physics.iisc.ernet.in/pocketdepth/ (accessed December 22, 2007).

[21] eHiTS: Electronic High Throughput Screening. http://www.simbiosys.ca/ehits/ehits_documents.html (accessed January 11, 2008).

[22] RCSB Protein Data Bank. http://www.rcsb.org/pdb/home/home.do (accessed November 25, 2007).

[23] UniProtKB/Swiss-Prot. http://www.ebi.ac.uk/swissprot/ (accessed December 7, 2007).

[24] Ezziane Z. In Intelligent Computing Everywhere; Schuster, Ed.; Springer Verlag: Heidelberg, 2007, pp. 195-209.

[25] Ezziane Z. Nanotechnology, 2006, 17, 27.

[26] Ezziane Z. In The First International Conference on Drug Design and Discovery, Abstracts of the $1^{\text {st }}$ International Conference on Drug Design and Discovery, Dubai, February 4-7, 2008. 